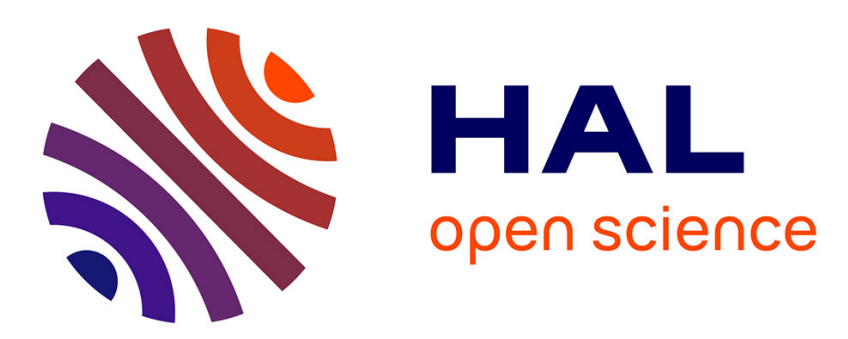

\title{
A novel control design for delayed teleoperation based on delay-scheduled Lyapunov-Krasovskii functionals
}

Bo Zhang, Alexandre Kruszewski, Jean-Pierre Richard

\section{To cite this version:}

Bo Zhang, Alexandre Kruszewski, Jean-Pierre Richard. A novel control design for delayed teleoperation based on delay-scheduled Lyapunov-Krasovskii functionals. International Journal of Control, 2014, 10.1080/00207179.2014.883464 . hal-00939507

\section{HAL Id: hal-00939507 \\ https://hal.inria.fr/hal-00939507}

Submitted on 30 Jan 2014

HAL is a multi-disciplinary open access archive for the deposit and dissemination of scientific research documents, whether they are published or not. The documents may come from teaching and research institutions in France or abroad, or from public or private research centers.
L'archive ouverte pluridisciplinaire HAL, est destinée au dépôt et à la diffusion de documents scientifiques de niveau recherche, publiés ou non, émanant des établissements d'enseignement et de recherche français ou étrangers, des laboratoires publics ou privés. 


\title{
RESEARCH ARTICLE
}

\section{A novel control design for delayed teleoperation based on delay-scheduled Lyapunov-Krasovskii functionals}

\author{
Bo Zhang $^{a *}$, Alexandre Kruszewski $^{a}$ and Jean-Pierre Richard ${ }^{a b}$ \\ ${ }^{a}$ Université Lille Nord de France, Ecole Centrale de Lille - 59651 Villeneuve d'Ascq, France and \\ LAGIS, CNRS UMR 8219, Laboratoire d'Automatique, Génie Informatique et Signal; ${ }^{b}$ INRIA NON-A
}

(v3.5 released August 2012)

\begin{abstract}
This paper addresses the controller design problem for bilateral teleoperation over unreliable networks. The stability and tracking performance analysis are presented for a novel force-reflecting emulator control scheme. The performance (stability, synchronization, transparency) is guaranteed by $H_{\infty}$ control theory and delayscheduled Lyapunov-Krasovskii functionals (LKF), which could improve the existing stability criterion. The design is achieved by using Linear Matrix Inequality (LMI) optimization. For the simulation, firstly, numerical examples are given to demonstrate the effectiveness and benefits of the delay-scheduled LKF-based stability results; secondly, the proposed controller design solution is illustrated by various simulations and compared with other recent approaches under different working conditions, e.g. abrupt tracking motion and wall contact.
\end{abstract}

Keywords: teleoperation system; delay-scheduled Lyapunov-Krasovskii functionals; Linear Matrix Inequality; asymmetric time-varying delays; $H_{\infty}$ control; position/force tracking

\section{Introduction}

Teleoperation systems extend the human manipulative capabilities to a remote environment. A typical teleoperation system is composed of the human operator, the master haptic-interface robot, the communication medium, the slave robot and the environment. In addition to the unilateral case (in which the motion or force information of the master robot handled by the human operator are transmitted only from the master to the slave), bilateral teleoperation is a closed-loop structure including the backward transmission from the slave to the master. It is a challenging area with many traditional or potential applications, such as telesurgery, undersea and space exploration, nuclear reactor control (Anderson and Spong 1989, Hua and Liu 2010). Long-range or flexible communication links such as the Internet or Wireless 802.11 networks are extremely interesting in such contexts. However, their unreliability and variations in the Quality of Service (QoS) introduce additional, complex dynamics (Chopra et al. 2008, Zampieri 2008) that can be represented by time-varying delays (Kruszewski et al. 2011). Because delays have a strong influence on the system performance (Richard 2003), they must be considered at the very stage of teleoperation control design.

Passivity-based control methods have been presented to address the stability of teleoperation systems with variable delays (Chopra et al. 2008). The survey (Nuño et al. 2011) revisits many passivity-based controllers for bilateral teleoperation system, including the scattering and wave variables. Besides, based on the energy and power considerations, time domain passivity control (Ryu et al. 2005, Ye et al. 2009) without the transformation of wave variables have been proposed

*Corresponding author. Email: bo.zhang@ec-lille.fr 
recently. Overall, the latest passivity-based results can resolve the stabilization problem under time-varying delays. However, the system performance (the position/force tracking) could still be improved. Additionally, the passivity method requires the human input force and the slave environment force to satisfy the passive condition, which introduces practical difficulties.

Various other control strategies have been proposed for a non-passive environment, in which additional estimation (e.g. force) or transformation (e.g. impedance) becomes necessary, we can mention several results depending on the nature of delays (the readers can refer to the survey (Arcara and Melchiorri 2002) to obtain various control strategies):

- Constant and/or symmetric delays: in (Garcia-Valdovinos et al. 2007, Shahbazi et al. 2010), it was proposed a control architecture based on the sliding mode approach; in (Hou et al. 2010), a half closed-loop framework to construct the bilateral control system with force feedback; in (Daly and Wang 2010), a position-force teleoperation algorithm based on the force estimation to handle nonlinear teleoperation.

- Time-varying delays: in (Hua and Liu 2011), it was proposed an exponentially stable scheme with or without the velocity signal; in (Delgado and Barreiro 2009), a 2-channel position error architecture by Lyapunov-Krasovskii and frequencial techniques; in (Natori et al. 2010), a compensation algorithm with network and communication disturbance observer (note that, contrarily to other compensation approaches like predictors (Smith and Hashtrudi-Zaad 2005, Witrant et al. 2007), this last does not require time delay models); in (Polushin et al. 2007), a forcereflecting teleoperation to improve the system performance. However, for these approaches, the synchronization or transparency of the teleoperation system is still hard to guarantee.

Apart from the stability of teleoperation systems under time-varying delays, there are also two kinds of performance for bilateral teleoperation, which are still open problems for bilateral teleoperation design (Chopra et al. 2006):

- Position tracking (or position coordination): the slave robot should follow the motion of the master robot maneuvered by the human operator.

- Force tracking (or force coordination): the environmental force acting on the slave (when it contacts the external environment) should be accurately transmitted in real-time to the master. This can be achieved by the force-reflecting, in which the human operator feels haptic sensations as if he or she is actually present at the slave side.

The present work addresses this last open problem under time-varying delays. We generalize a previous result on a bilateral state feedback control structure (Zhang et al. 2011) to allow position tracking under asymmetric and time-varying delays. From this result, we present a new control structure and prove the stability of the closed-loop system by Lyapunov approaches, especially, Lyapunov-Krasovskii functionals (LKF), which can be solved by Linear Matrix Inequalities (LMI, see (Fridman 2006) and the references therein). However, differently from (He et al. 2007, Shao 2009), we will present delay-scheduled Lyapunov-Krasovskii functionals, which could improve the delay-dependent stability criterion and be more convenient to the stabilization problem.

For the performance consideration, $H_{\infty}$ control theory will be used in order to make the position error (from master to slave) converges to a small region (Fridman and Shaked 2001, Xu et al. 2006, Zhang and Han 2008). Finally, the characteristic of the control law will ensure a good force tracking (from slave to master).

This paper is organized as follows: Section 2 introduces delay-scheduled Lyapunov-Krasovskii functionals, and several resulting stability theorems. Problems and assumptions are presented in Section 3. In Section 4, The novel force-reflecting control structure with force feedback is designed by using delay-scheduled LKF and $H_{\infty}$ control. Numerical examples to demonstrate the effectiveness and merits of delay-scheduled LKF-based stability results are given in Section 5 , and then simulation results and comparisons with other design techniques for teleoperation are presented in Section 6. Finally we conclude our works in Section 7. 


\section{Delay-scheduled LKF-based stability}

Teleoperation system are systems with time varying delay. There exist a lot of results about this class of models and one could note the ones presented in (Park et al. 2011). Despite the fact that the results of (Park et al. 2011) are one of the less conservative for time varying delays, they are not adapted for the teleoperation problem since they do not allow the control design. This section if devoted to provide some conditions for the stability of linear time varying delay systems which can be adapted to multiple delays and control design with $H_{\infty}$ performance guarantee. They are based on the Lyapunov function of (Shao 2009), the free weighting matrix and the polytopic approach for time-varying delays. For simplicity reasons, these results are presented in the one time varying delay case. Nevertheless, the results can be easily extended. Let consider the following model:

$$
\left(\Sigma_{1}\right)\left\{\begin{array}{l}
\dot{x}(t)=A_{0} x(t)+A_{1} x(t-\tau(t))+B w(t), \\
z(t)=C x(t), \\
x\left(t_{0}+\theta\right)=\phi(\theta), \quad \dot{x}\left(t_{0}+\theta\right)=\dot{\phi}(\theta), \quad \theta \in\left[-h_{2}, 0\right] .
\end{array}\right.
$$

Here, $x(t) \in \mathbf{R}^{n}, w(t) \in \mathbf{R}^{l}$ is some exogenous disturbance signals, while $z(t) \in \mathbf{R}^{q}$ is the objective control output. $\phi(\theta)$ is the initial state function, and $\tau(t) \in\left[h_{1}, h_{2}\right], h_{1}>0$, is the time-varying delay. $A_{0}, A_{1}, B$ and $C$ are constant matrices.

We consider the delay with the constraints on the derivative: $\dot{\tau}(t) \leqslant \mu, \mu \leqslant 1$. The LyapunovKrasovskii functional presented in (Shao 2009) is considered:

$$
\begin{aligned}
& V(x(t), \dot{x}(t))=x(t)^{T} P x(t)+\int_{t-\tau(t)}^{t} x(s)^{T} Q x(s) d s+\int_{t-h_{2}}^{t} x(s)^{T} S_{a} x(s) d s+\int_{t-h_{1}}^{t} x(s)^{T} S x(s) d s \\
& +\int_{-h_{1}}^{0} \int_{t+\theta}^{t} \dot{x}(s)^{T} R \dot{x}(s) d s d \theta+\int_{-h_{2}}^{-h_{1}} \int_{t+\theta}^{t} \dot{x}(s)^{T} R_{a} \dot{x}(s) d s d \theta .
\end{aligned}
$$

According to $H_{\infty}$ control theory, the performance will be studied by checking $H_{\infty}$ performance criterion $J(w)<0$ for a positive scalar $\gamma$ :

$$
J(w)=\int_{0}^{\infty}\left(z(t)^{T} z(t)-\gamma^{2} w(t)^{T} w(t)\right) d t<0 .
$$

Theorem 2.1: $\quad$ Suppose there exist $n \times n$ symmetric matrices $P>0, Q>0, R>0, S>0$, $S_{a}>0, R_{a}>0$, some matrices $P_{2}, P_{3}, P_{4}, Y_{1}, Y_{2}, Y_{3}, N_{1}, N_{2}, N_{3}$, and a positive scalar $\gamma$, such that LMI condition (4) with notations (5) is feasible. Then, the system (1) is asymptotically stable with $J(w)<0$ (3) for the time-varying delay $\tau(t)$.

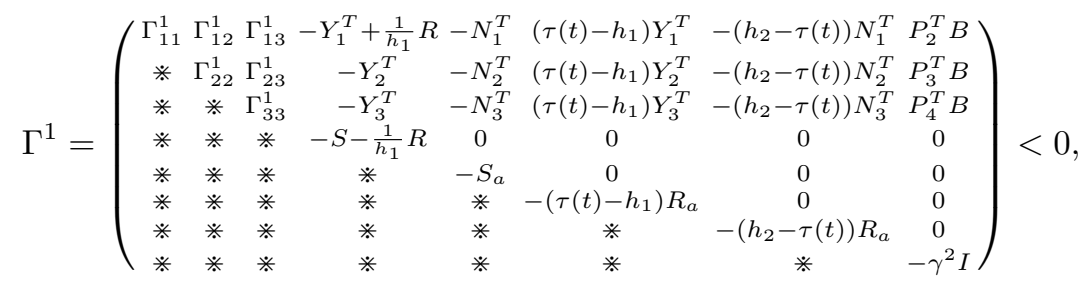

$$
\begin{aligned}
& \Gamma_{11}^{1}=Q+S+S_{a}-\frac{1}{h_{1}} R+A_{0}^{T} P_{2}+P_{2}^{T} A_{0}+C^{T} C, \quad \Gamma_{12}^{1}=P-P_{2}^{T}+A_{0}^{T} P_{3}, \\
& \Gamma_{13}^{1}=Y_{1}^{T}+N_{1}^{T}+A_{0}^{T} P_{4}+P_{2}^{T} A_{1}, \quad \Gamma_{22}^{1}=-P_{3}-P_{3}^{T}+h_{1} R+\left(h_{2}-h_{1}\right) R_{a}, \\
& \Gamma_{23}^{1}=Y_{2}^{T}+N_{2}^{T}-P_{4}+P_{3}^{T} A_{1}, \quad \Gamma_{33}^{1}=-(1-\mu) Q+Y_{3}+Y_{3}^{T}+N_{3}+N_{3}^{T}+A_{1}^{T} P_{4}+P_{4}^{T} A_{1} .
\end{aligned}
$$

Proof Considering $V(x(t), \dot{x}(t))$ in (2) and substituting for $z(t)$, one obtains: 


$$
\begin{aligned}
& \dot{V}(x(t), \dot{x}(t))+z(t)^{T} z(t)-\gamma^{2} w(t)^{T} w(t) \\
& \leqslant x(t)^{T}\left(Q+S+S_{a}+C^{T} C\right) x(t)+\dot{x}(t)^{T} P x(t)+x(t)^{T} P \dot{x}(t) \\
& -x(t-\tau(t))^{T}(1-\mu) Q x(t-\tau(t))-x\left(t-h_{1}\right)^{T} S x\left(t-h_{1}\right)-x\left(t-h_{2}\right)^{T} S_{a} x\left(t-h_{2}\right) \\
& +\dot{x}(t)^{T}\left[h_{1} R+\left(h_{2}-h_{1}\right) R_{a}\right] \dot{x}(t)-\int_{t-h_{1}}^{t} \dot{x}(s)^{T} R \dot{x}(s) d s-\int_{t-h_{2}}^{t-h_{1}} \dot{x}(s)^{T} R_{a} \dot{x}(s) d s \\
& -\gamma^{2} w(t)^{T} w(t) .
\end{aligned}
$$

From (Zhang et al. 2011), we can see that, $J(w)<0$ holds if $(6)$ is negative. We apply Jensen's inequality ( $\mathrm{Gu}$ et al. 2003):

$$
\begin{gathered}
-\int_{t-h_{1}}^{t} \dot{x}(s)^{T} R \dot{x}(s) d s \leqslant-\int_{t-h_{1}}^{t} \dot{x}(s)^{T} d s \frac{1}{h_{1}} R \int_{t-h_{1}}^{t} \dot{x}(s) d s \\
-\int_{t-h_{2}}^{t-h_{1}} \dot{x}(s)^{T} R_{a} \dot{x}(s) d s=-\int_{t-h_{2}}^{t-\tau(t)} \dot{x}(s)^{T} R_{a} \dot{x}(s) d s-\int_{t-\tau(t)}^{t-h_{1}} \dot{x}(s)^{T} R_{a} \dot{x}(s) d s \\
\leqslant-\left(\tau(t)-h_{1}\right) v_{1}^{T}(\tau(t)) R_{a} v_{1}(\tau(t))-\left(h_{2}-\tau(t)\right) v_{2}^{T}(\tau(t)) R_{a} v_{2}(\tau(t)),
\end{gathered}
$$

where:

$$
v_{1}(\tau(t))=\frac{1}{\tau(t)-h_{1}} \int_{t-\tau(t)}^{t-h_{1}} \dot{x}(s) d s, \quad v_{2}(\tau(t))=\frac{1}{h_{2}-\tau(t)} \int_{t-h_{2}}^{t-\tau(t)} \dot{x}(s) d s,
$$

and then, we add free weighting matrices $P_{2}, P_{3}, P_{4}, Y_{1}, Y_{2}, Y_{3}, N_{1}, N_{2}, N_{3}$, and the following expressions into (6):

$$
\begin{aligned}
& 0=2\left[x(t)^{T} Y_{1}^{T}+\dot{x}(t)^{T} Y_{2}^{T}+x(t-\tau(t))^{T} Y_{3}^{T}\right]\left[x(t-\tau(t))+\left(\tau(t)-h_{1}\right) v_{1}(\tau(t))-x\left(t-h_{1}\right)\right], \\
& 0=2\left[x(t)^{T} N_{1}^{T}+\dot{x}(t)^{T} N_{2}^{T}+x(t-\tau(t))^{T} N_{3}^{T}\right]\left[x(t-\tau(t))-\left(h_{2}-\tau(t)\right) v_{2}(\tau(t))-x\left(t-h_{2}\right)\right], \\
& 0=2\left[x(t)^{T} P_{2}^{T}+\dot{x}(t)^{T} P_{3}^{T}+x(t-\tau(t))^{T} P_{4}^{T}\right]\left[A_{0} x(t)+B w(t)+A_{1} x(t-\tau(t))-\dot{x}(t)\right] .
\end{aligned}
$$

In order to obtain LMI condition, the notation as:

$$
\eta(t)=\operatorname{col}\left\{x(t), \dot{x}(t), x(t-\tau(t)), x\left(t-h_{1}\right), x\left(t-h_{2}\right), v_{1}(\tau(t)), v_{2}(\tau(t)), w(t)\right\},
$$

leading to:

$$
\dot{V}(x(t), \dot{x}(t))+z(t)^{T} z(t)-\gamma^{2} w(t)^{T} w(t) \leq \eta(t)^{T} \Gamma^{1} \eta(t)<0,
$$

provides that the LMI (4) is feasible.

Remark 1: Because (4) is linear with regard to $\tau(t)$, Theorem 2.1 conditions can be solved using polytopic approaches. The result lies in a set of two LMIs conditions, one at $\tau(t)=h_{1}$ and another at $\tau(t)=h_{2}$. Indeed, in these two LMIs, rows/columns full of 0 can be discarded.

When the information of the time derivative of delay is unknown i.e. $\mu=1$, it is possible to get a simplified version of Theorem 2.1.

Corollary 2.2: $\quad$ Suppose there exist $n \times n$ symmetric matrices $P>0, R>0, S>0, S_{a}>0$, $R_{a}>0$, some matrices $P_{2}, P_{3}, Y_{1}, Y_{2}$, and a positive scalar $\gamma$, such that LMI condition (13) with notations (14) is feasible. Then, the system (1) is asymptotically stable with $J(w)<0$ (3) for the time-varying delay $\tau(t)$. 


$$
\begin{aligned}
& \Gamma^{2}=\left(\begin{array}{ccccccc}
\Gamma_{11}^{2} & \Gamma_{12}^{2} & -Y_{1}^{T}+\frac{1}{h_{1}} R+P_{2}^{T} A_{1} & Y_{1}^{T} & \Gamma_{15}^{2} & \left(h_{2}-\tau(t)\right) Y_{1}^{T} & P_{2}^{T} B \\
* & \Gamma_{22}^{2} & -Y_{2}^{T}+P_{3}^{T} A_{1} & Y_{2}^{T} & \Gamma_{25}^{2} & \left(h_{2}-\tau(t)\right) Y_{2}^{T} & P_{3}^{T} B \\
* & * & -S-\frac{1}{h_{1}} R & 0 & 0 & 0 & 0 \\
* & * & * & -S_{a} & 0 & 0 & 0 \\
* & * & * & * & -\left(\tau(t)-h_{1}\right) R_{a} & 0 & 0 \\
* & * & * & * & * & -\left(h_{2}-\tau(t)\right) R_{a} & 0 \\
* & * & * & * & * & * & *
\end{array}\right)<0, \\
& \Gamma_{11}^{2}=S+S_{a}-\frac{1}{h_{1}} R+A_{0}^{T} P_{2}+P_{2}^{T} A_{0}+C^{T} C, \quad \Gamma_{12}^{2}=P-P_{2}^{T}+A_{0}^{T} P_{3}, \\
& \Gamma_{15}^{2}=\left(\tau(t)-h_{1}\right) Y_{1}^{T}-\left(\tau(t)-h_{1}\right) P_{2}^{T} A_{1}, \\
& \Gamma_{22}^{2}=-P_{3}-P_{3}^{T}+h_{1} R+\left(h_{2}-h_{1}\right) R_{a}, \quad \Gamma_{25}^{2}=\left(\tau(t)-h_{1}\right) Y_{2}^{T}-\left(\tau(t)-h_{1}\right) P_{3}^{T} A_{1} .
\end{aligned}
$$

Proof The proof is similar to Theorem 2.1 with some differences:

- eliminating $Q$ in (2);

- introducing free weighting matrices and the expressions not as (10), but as follows:

$$
\begin{aligned}
& 0=2\left[x(t)^{T} P_{2}^{T}+\dot{x}(t)^{T} P_{3}^{T}\right]\left[A_{0} x(t)+B w(t)+A_{1} x\left(t-h_{1}\right)-\left(\tau(t)-h_{1}\right) A_{1} v_{1}(\tau(t))-\dot{x}(t)\right], \\
& 0=2\left[x(t)^{T} Y_{1}^{T}+\dot{x}(t)^{T} Y_{2}^{T}\right]\left[x\left(t-h_{2}\right)+\left(\tau(t)-h_{1}\right) v_{1}(\tau(t))+\left(h_{2}-\tau(t)\right) v_{2}(\tau(t))-x\left(t-h_{1}\right)\right] ;
\end{aligned}
$$

- using notation:

$$
\eta(t)=\operatorname{col}\left\{x(t), \dot{x}(t), x\left(t-h_{1}\right), x\left(t-h_{2}\right), v_{1}(\tau(t)), v_{2}(\tau(t)), w(t)\right\} ;
$$

Then the result follows: $\dot{V}(x(t), \dot{x}(t))+z(t)^{T} z(t)-\gamma^{2} w(t)^{T} w(t) \leq \eta(t)^{T} \Gamma^{2} \eta(t)<0$.

Remark 2 : $\quad$ Compared to (He et al. 2007, Shao 2009, Park et al. 2011), the Jensen's inequality is used only one time which could reduce the conservatism of the conditions. It is rather hard to prove it due to the number of bounding techniques used. In order to get tractable conditions for the control design which is not possible if we follow (Park et al. 2011), we have modified the expressions used with the free weighting matrix techniques (see (10) and (15)).

We will also use the following corollary that considers Theorem 2.1 in the delay-free case (without $\left.A_{1} x(t-\tau(t))\right)$ :

$$
\left(\Sigma_{2}\right)\left\{\begin{array}{l}
\dot{x}(t)=A_{0} x(t)+B w(t), \\
z(t)=C x(t) .
\end{array}\right.
$$

Corollary 2.3: $\quad$ Suppose there exist a $n \times n$ symmetric matrice $P>0$, some matrices $P_{2}, P_{3}$, and a positive scalar $\gamma$, such that LMI condition (18) with the notation (19) is feasible. Then, the system (17) is asymptotically stable with $J(w)<0$ (3).

$$
\begin{gathered}
\Gamma^{3}=\left(\begin{array}{ccc}
\Gamma_{11}^{3} & P-P_{2}^{T}+A_{0}^{T} P_{3} & P_{2}^{T} B \\
* & -P_{3}-P_{3}^{T} & P_{3}^{T} B \\
* & * & -\gamma^{2} I
\end{array}\right)<0, \\
\Gamma_{11}^{3}=A_{0}^{T} P_{2}+P_{2}^{T} A_{0}+C^{T} C .
\end{gathered}
$$

\section{Problem formulation of delayed teleoperation}

Let us give a short recall of the bilateral state feedback control scheme given in (Zhang et al. 2011). Note that ignoring the details of master and slave controllers, this scheme corresponds to 


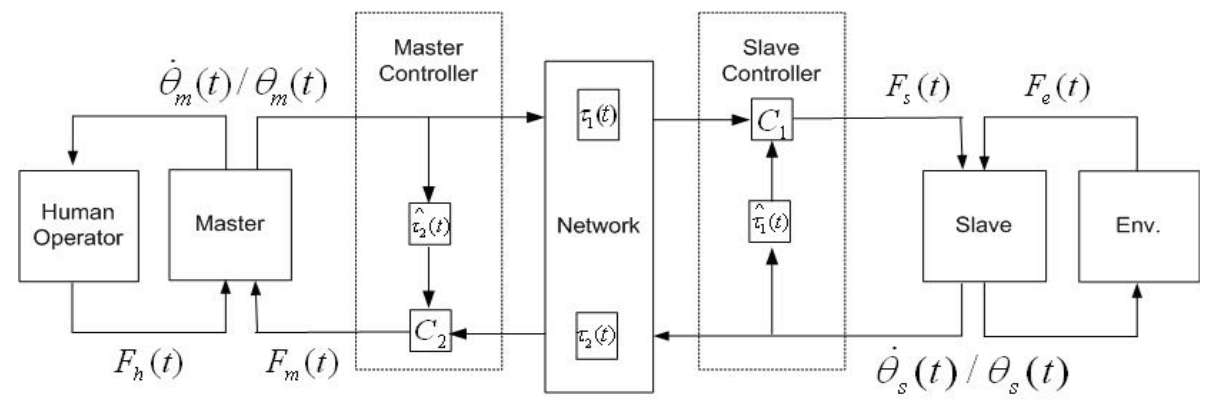

Figure 1. Bilateral state feedback control scheme.

a general teleoperation system with five entities (human operator, master robot, communication medium, slave robot, environment). In this general scheme (Arcara and Melchiorri 2002):

- Master controller and slave controller are the global controllers we should design so to ensure both the asymptotic stability of the whole system and the position/force tracking between the master and the slave (in Figure 1, $C_{1}$ and $C_{2}$ are the global controllers that we should design).

- $F_{m}(t)$ and $F_{s}(t)$ are the actuated inputs of the master and of the slave.

- $F_{h}(t)$ and $F_{e}(t)$ are the forces of the human operator and of the environment. Note that these forces are considered as the disturbances and the global controllers have to minimize their effects on the output tracking error.

- $\tau_{1}(t)$ (from the master to the slave) and $\tau_{2}(t)$ (from the slave to the master) are the delays (here, time-varying and asymmetric) resulting from the communication, access time, and packet loss effects (Kruszewski et al. 2011).

- $\dot{\theta}_{m}(t) / \theta_{m}(t)$ and $\dot{\theta}_{s}(t) / \theta_{s}(t)$ are the velocities/positions of the master and the slave.

- The information transferred between the master and the slave can be the positions, the velocities or the estimated forces of the human operator and environment (in Figure 1, the velocities/positions of the master and the slave).

For state feedback controllers, the master and the slave are given by:

According to the general scheme, the following assumptions are made.

Assumption 3.1 The master and slave robots can be modeled as linear systems. It is reasonable to consider linear model for the two sides in many teleoperation problems because most the robots used are serial robots. These robot are easily linearized by choosing the right controller with a linear reference model as soon as the trajectories respect the systems boundaries (control saturation, joints not close to a physical limits or singularities ...). The use of the calculated torque control (for low friction) or sliding mode control (for high friction) are examples of how it could be done. Whatever the choice made for this linearization, it will be considered in the following that both sides are disturbed and the control will be design to get good performances despite those disturbances. The Master and Slave dynamics are given by:

$$
\begin{aligned}
\left(\Sigma_{m}\right) \quad \ddot{\theta}_{m}(t) & =\left(A_{m}-B_{m} K_{0}^{m}\right) \dot{\theta}_{m}(t)+B_{m}\left(F_{m}(t)+F_{h}(t)\right), \\
\left(\Sigma_{s}\right) & \ddot{\theta}_{s}(t)=\left(A_{s}-B_{s} K_{0}^{s}\right) \dot{\theta}_{s}(t)+B_{s}\left(F_{s}(t)+F_{e}(t)\right),
\end{aligned}
$$

where $K_{0}^{m}$ and $K_{0}^{s}$ are known speed feedbacks, $A_{m}, B_{m}$ and $A_{s}, B_{s}$ are the constant matrices.

Assumption 3.2 The communication delays are bounded, $\tau_{1}(t), \tau_{2}(t) \in\left[h_{1}, h_{2}\right], h_{1}>0$, $h_{2}<\infty$. The communication over network such as the internet are not bounded but in all teleoperation process, a delay limit is chosen to avoid dangerous situation, if the delay goes out of this bound, the teleoperation stops.

Assumption 3.3 Master and slave clocks are perfectly synchronized and the data packets are timestamped with the sending time. This allows the Master (Slave) to compute the Slave-to-Master (Master-to-Slave) delay: $\hat{\tau}_{1}(t)=\tau_{1}(t), \hat{\tau}_{2}(t)=\tau_{2}(t)$. The clocks of both sides can be synchronized. Even if it is impossible to get perfect synchronization, 
it is possible to get a close enough result to consider as perfect. It is needed to get an error of synchronization negligible with respect to the delay. For small networks with a low traffic, it could be achieved using a network time protocol as in (Kruszewski et al. 2011). If the network as a lot of nodes/high traffic the use of GPS clock could solve the problem as in (Jiang et al. 2008).

Assumption 3.4 The external forces $F_{h}(t)$ and $F_{e}(t)$ are accessible. The external forces can be obtained either by using obtained sensors (strain gauge) or a unknown input observer. The choice between the two solutions depends only on the degree of fidelity of the models. The result obtained using Strain gauges is independent on the model fidelity but can be tricky to adapt on the mechanical structure of the robot (especially the master which is small). The unknown input observer technique is only suitable if the model is really good and there are not too much friction. Usually, strain gauges are used in the slave side and observer in the master side.

\section{Novel force-reflecting emulator control scheme}

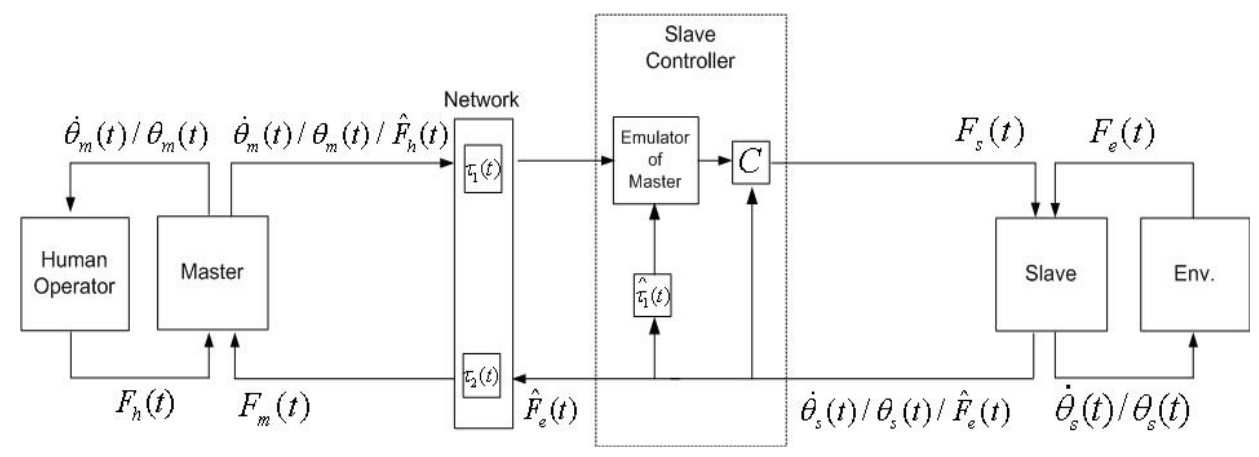

Figure 2. Novel force-reflecting emulator control scheme.

Let consider the force-reflecting control structure depicted in Figure 2. The two main differences with the previously presented structure are the direct force feedback $F_{m}(t)=F_{e}\left(t-\tau_{2}\right)$ ensuring a perfect telepresence and the presence of an estimator called "emulator" which estimates the current state of the master $x_{e}(t) \approx x_{m}(t)$ i.e. without delay. Considering this structure reduces the number of delay involved in each loop. Because LKF techniques are more conservative when more delays are involved and because delays are generally bad for closed loop systems, this structure helps to get better guaranteed performances from the position tracking point of view. Figure 2. The two main differences with the previously presented structure are the direct force feedback $F_{m}(t)=F_{e}\left(t-\tau_{2}\right)$ ensuring a perfect telepresence and the presence of an estimator called "emulator" which estimates the current state of the master $x_{e}(t) \approx x_{m}(t)$ i.e. without delay. Considering this structure reduces the number of delay involved in each loop. Because LKF techniques are more conservative when more delays are involved and because delays are generally bad for closed loop systems, this structure helps to get better guaranteed performances from the position tracking point of view.

\subsection{Slave controller description}

The Master emulator presented in Figure 2 is a distant copy of the master dynamics. The current Master state is estimated using an observer-like structure where $\dot{\theta}_{e}(t)$ and $\theta_{e}(t)$ are estimated values of the current velocity/position of the Master $\dot{\theta}_{m}(t)$ and $\theta_{m}(t)$. The main goal of this estimator is to 'remove' the Master state delay on the Slave side. The model of the emulator is as follow: 


$$
\begin{gathered}
\left(\Sigma_{e}\right) \quad \ddot{\theta}_{e}(t)=\left(A_{m}-B_{m} K_{0}^{m}\right) \dot{\theta}_{e}(t)-B_{m} Y_{e}(t)+B_{m}\left(F_{e}\left(t-\tau_{1}(t)\right)+F_{h}\left(t-\tau_{1}(t)\right)\right) . \\
Y_{e}(t)=L\left(\begin{array}{c}
\dot{\theta}_{e}\left(t-\tau_{1}(t)\right) \\
\dot{\theta}_{m}\left(t-\tau_{1}(t)\right) \\
\theta_{e}\left(t-\tau_{1}(t)\right)-\theta_{m}\left(t-\tau_{1}(t)\right)
\end{array}\right) .
\end{gathered}
$$

Remark 3: Note that the emulator has the same equation as the Master. The only difference is the delay on th inputs and the presence to the synchronization term $Y_{e}$. Instead of using the classical Luenberger equation, the correction term $Y_{e}$ act as a force for this dynamical model. This choice is made to avoid unatural estimated trajectory that an observer could generate when synchronizing the state and the estimation.

The controller $C$ role is to ensure the position tracking between the slave and the emulator state (estimation of the current Master state). It is a simple state feedback with the following equation:

$$
F_{s}(t)=-K\left(\begin{array}{c}
\dot{\theta}_{s}(t) \\
\dot{\theta}_{e}(t) \\
\theta_{s}(t)-\theta_{e}(t)
\end{array}\right)
$$

\subsection{Controller design}

Our objective is to provide a controller design algorithm for the emulator of master and the controller $C$, to achieve the stability of the whole system with the position/force tracking. It is done by following three steps: First the master emulator is designed to achieve a correct estimation of the current master state, then the controller $C$ is designed to ensure that the slave tracks as best as possible the master emulator position. Finally a global analysis is performed to check if the performances are good enough.

\subsubsection{Master-emulator synchronization}

First of all, we will design the gain $L$ to ensure a robust estimation of the Master state by the emulator. This is one by minimizing the $H_{\infty}$ gain between the external inputs, with respect to the Master-Emulator subsystem, $F_{e}$ and $F_{h}$ the estimation error $\theta_{e}(t)-\theta_{m}(t)$. This problem can be represented as a stabilization problem involving the following models:

$$
\left(\Sigma_{m e}\right)\left\{\begin{array}{l}
\dot{x}_{m e}(t)=A_{m e}^{0} x_{m e}(t)+A_{m e}^{1} x_{m e}\left(t-\tau_{1}(t)\right)+B_{m e} w_{m e}(t), \\
z_{m e}(t)=C_{m e} x_{m e}(t)
\end{array}\right.
$$

where:

$$
x_{m e}(t)=\left(\begin{array}{c}
\dot{\theta}_{\theta}(t) \\
\dot{\theta}_{m}(t) \\
\theta_{e}(t)-\theta_{m}(t)
\end{array}\right), \quad w_{m e}(t)=\left(\begin{array}{c}
F_{e}\left(t-\tau_{1}(t)\right)+F_{h}\left(t-\tau_{1}(t)\right) \\
F_{m}(t)+F_{h}(t)
\end{array}\right), \quad z_{m e}(t)=\left(\theta_{e}(t)-\theta_{m}(t)\right) .
$$

Then:

$$
\begin{aligned}
A_{m e}^{0} & =\left(\begin{array}{ccc}
A_{m}-B_{m} K_{0}^{m} & 0 & 0 \\
0 & A_{m}-B_{m} K_{0}^{m} & 0 \\
1 & -1 & 0
\end{array}\right), \quad A_{m e}^{1}=\left(\begin{array}{ccc}
-B_{m} L_{1} & -B_{m} L_{2} & -B_{m} L_{3} \\
0 & 0 & 0 \\
0 & 0 & 0 \\
0
\end{array}\right), \\
B_{m e} & =\left(\begin{array}{cc}
B_{m} & 0 \\
0 & B_{m} \\
0 & 0
\end{array}\right)=\left(\begin{array}{ll}
B_{m e}^{1} B_{m e}^{2}
\end{array}\right), \quad C_{m e}=\left(\begin{array}{lll}
0 & 0 & 1
\end{array}\right) .
\end{aligned}
$$

From (26) and $L=\left(\begin{array}{lll}L_{1} & L_{2} & L_{3}\end{array}\right)$, one has: $A_{m e}^{1}=-B_{m e}^{1} L$.

Design Goal 4.1 By $H_{\infty}$ control design, we can obtain the minimum value of $\gamma$ (as $\gamma_{\text {min }}$ ), and the bound $\sup _{w}\left(\left\|z_{m e}(t)\right\|_{2} /\left\|w_{m e}(t)\right\|_{2}\right)<\gamma_{\min }$ (Tadmor 2000). Thus, the position deviation $z_{m e}(t)$ can be minimized under exogenous disturbance input $w_{m e}(t)$ : the smaller $\gamma_{\text {min }}$, the better $H_{\infty}$ synchronization performance. 
Thus, the following theorem is obtained by using Corollary 2.2 (Theorem 2.1 can also be applied if the information of $\dot{\tau}(t)$ is known).

Theorem 4.2: $\quad$ Suppose there exist symmetric matrices $P>0, R>0, S>0, S_{a}>0, R_{a}>0$, some matrices $P_{2}, Y_{1}, Y_{2}, M$, and positive scalars $\gamma$ and $\xi$, such that LMI condition (28) with notations (29) is feasible. Then, the system (24) is asymptotically stable with $J(w)<0$ (3) for the time-varying delay $\tau_{1}(t)$ and the following emulator control gain:

$$
\begin{aligned}
& L=M P_{2}^{-1} . \\
& \Gamma^{4}=\left(\begin{array}{cccccccc}
\Gamma_{11}^{4} \Gamma_{12}^{4}-Y_{1}^{T}+\frac{1}{h_{1}} R-B_{m e}^{1} M & Y_{1}^{T} & \Gamma_{15}^{4} & \left(h_{2}-\tau_{1}(t)\right) Y_{1}^{T} & B_{m e} & P_{2}^{T} C_{m e}^{T} \\
* & \Gamma_{22}^{4} & -Y_{2}^{T}-\xi B_{m e}^{1} M & Y_{2}^{T} & \Gamma_{25}^{4} & \left(h_{2}-\tau_{1}(t)\right) Y_{2}^{T} & \xi B_{m e} & 0 \\
* & * & -S-\frac{1}{h_{1}} R & 0 & 0 & 0 & 0 & 0 \\
* & * & * & -S_{a} & 0 & 0 & 0 & 0 \\
* & * & * & *\left(\tau_{1}(t)-h_{1}\right) R_{a} & 0 & 0 & 0 \\
* & * & * & * & * & -\left(h_{2}-\tau_{1}(t)\right) R_{a} & 0 & 0 \\
* & * & * & * & * & * & * & * \\
* & * & * & * & * & * & * & 0 \\
* & * & * & * & &
\end{array}\right)<0, \\
& \Gamma_{11}^{4}=S+S_{a}-\frac{1}{h_{1}} R+P_{2}^{T} A_{m e}^{0}{ }^{T}+A_{m e}^{0} P_{2}, \quad \Gamma_{12}^{4}=P-P_{2}+\xi P_{2}^{T} A_{m e}^{0}{ }^{T}, \\
& \Gamma_{15}^{4}=\left(\tau_{1}(t)-h_{1}\right) Y_{1}^{T}+\left(\tau_{1}(t)-h_{1}\right) B_{m e}^{1} M, \\
& \Gamma_{22}^{4}=-\xi P_{2}-\xi P_{2}^{T}+h_{1} R+\left(h_{2}-h_{1}\right) R_{a}, \quad \Gamma_{25}^{4}=\left(\tau_{1}(t)-h_{1}\right) Y_{2}^{T}+\xi\left(\tau_{1}(t)-h_{1}\right) B_{m e}^{1} M .
\end{aligned}
$$

Proof We use Corollary 2.2 on system (24), a series of steps is made to deal with nonlinear matrix terms $P_{2}^{T} B_{m e}^{1} L$ and $P_{3}^{T} B_{m e}^{1} L$ (see also Fridman and Shaked (2001)):

- multiplying $\Gamma^{2}$ by $\operatorname{diag}\left\{P_{2}^{-T}, \ldots, P_{2}^{-T}\right\}$ at the left side, by $\operatorname{diag}\left\{P_{2}^{-1}, \ldots, P_{2}^{-1}\right\}$ at the right side;

- choosing $P_{3}=\xi P_{2}$;

- defining $M=L P_{2}$;

- applying Schur formula, then the result follows.

\subsubsection{Emulator-slave synchronization}

The position tracking between the master and the emulator of master has been achieved. The position tracking between the emulator of master and the slave will be assured by the controller $C$. The model of the system containing the emulator of master, the controller $C$ and the slave, is given as follows:

$$
\left(\Sigma_{e s}\right)\left\{\begin{array}{l}
\dot{x}_{e s}(t)=A_{e s} x_{e s}(t)+B_{e s} w_{e s}(t), \\
z_{e s}(t)=C_{e s} x_{e s}(t),
\end{array}\right.
$$

where the input of the emulator $Y_{e}(t)$ is considered as the exogenous disturbance signal:

$$
x_{e s}(t)=\left(\begin{array}{c}
\dot{\theta}_{s}(t) \\
\dot{\theta}_{e}(t) \\
\theta_{s}(t)-\theta_{e}(t)
\end{array}\right), \quad w_{e s}(t)=\left(\begin{array}{c}
F_{e}(t) \\
F_{e}\left(t-\tau_{1}(t)\right)+F_{h}\left(t-\tau_{1}(t)\right)-Y_{e}(t)
\end{array}\right), \quad z_{e s}(t)=\left(\theta_{s}(t)-\theta_{e}(t)\right) .
$$

So:

$$
A_{e s}=\left(\begin{array}{ccc}
A_{s}-B_{s} K_{0}^{s}-B_{s} K_{1} & -B_{s} K_{2} & -B_{s} K_{3} \\
0 & A_{m}-B_{m} K_{0}^{m} & 0 \\
1 & -1 & 0
\end{array}\right), \quad B_{e s}=\left(\begin{array}{cc}
B_{s} & 0 \\
0 & B_{m} \\
0 & 0
\end{array}\right)=\left(\begin{array}{ll}
B_{e s}^{1} & B_{e s}^{2}
\end{array}\right), \quad C_{e s}=\left(\begin{array}{lll}
0 & 0 & 1
\end{array}\right) .
$$

The controller gain $K$ should be calculated by LMI with the following transformation: 


$$
A_{e s}=\left(\begin{array}{ccc}
A_{s}-B_{s} K_{0}^{s} & 0 & 0 \\
0 & A_{m}-B_{m} K_{0}^{m} & 0 \\
1 & -1 & 0
\end{array}\right)+\left(\begin{array}{ccc}
-B_{s} K_{1} & -B_{s} K_{2} & -B_{s} K_{3} \\
0 & 0 & 0 \\
0 & 0 & 0
\end{array}\right)=A_{e s}^{0}-B_{e s}^{1} K .
$$

Similarly with the objective as Design Goal 4.1, we obtain the following theorem.

Theorem 4.3: $\quad$ Suppose there exist a symmetric matrice $P>0$, some matrices $P_{2}, W$, and positive scalars $\gamma$ and $\xi$, such that LMI condition (35) with notations (36) is feasible. Then, the system (30) is asymptotically stable with $J(w)<0$ (3) and with the control gain of the controller $C$ :

$$
\begin{aligned}
& K=W P_{2}^{-1} . \\
& \Gamma^{4}=\left(\begin{array}{cccc}
\Gamma_{11}^{4} & \Gamma_{12}^{4} & B_{e s} & P_{2}^{T} C_{e s}^{T} \\
* & \Gamma_{22}^{4} & \xi B_{e s} & 0 \\
* & * & -\gamma^{2} I & 0 \\
* & * & * & -I
\end{array}\right)<0, \\
& \Gamma_{11}^{4}=P_{2}^{T} A_{e s}^{0^{T}}+A_{e s}^{0} P_{2}-W^{T} B_{e s}^{1 T}-B_{e s}^{1} W, \\
& \Gamma_{12}^{4}=P-P_{2}+\xi P_{2}^{T} A_{e s}^{0^{T}}-\xi W^{T} B_{e s}^{1^{T}}, \quad \Gamma_{22}^{4}=-\xi P_{2}-\xi P_{2}^{T} .
\end{aligned}
$$

Proof We apply the system (30) and (33) in Corollary 2.3, and multiply $\Gamma^{3}$ by $\operatorname{diag}\left\{P_{2}^{-T}, \ldots, P_{2}^{-T}\right\}$ at the left side, by $\operatorname{diag}\left\{P_{2}^{-1}, \ldots, P_{2}^{-1}\right\}$ at the right side. Then, we define $P_{3}=\xi P_{2}, W=K P_{2}$. Finally, the theorem can be obtained by Schur formula.

\subsubsection{Global performance analysis}

By Theorem 4.2 and Theorem 4.3, the position tracking between the master, the emulator and the slave is ensured. Finally, the objective is to ensure the global stability of the whole system described by:

$$
\left(\Sigma_{m e s}\right)\left\{\begin{array}{l}
\dot{x}_{m e s}(t)=A_{m e s}^{0} x_{m e s}(t)+A_{m e s}^{1} x_{m e s}\left(t-\tau_{1}(t)\right)+B_{m e s} w_{m e s}(t) \\
z_{m e s}(t)=C_{m e s} x_{m e s}(t)
\end{array}\right.
$$

where:

$$
x_{m e s}(t)=\left(\begin{array}{c}
\dot{\theta}_{s}(t) \\
\dot{\theta}_{e}(t) \\
\dot{\theta}_{m}(t) \\
\theta_{s}(t)-\theta_{e}(t) \\
\theta_{e}(t)-\theta_{m}(t)
\end{array}\right), \quad w_{m e s}(t)=\left(\begin{array}{c}
F_{e}(t) \\
\left.F_{e}\left(t-\tau_{1}(t)\right)+F_{h}\left(t-\tau_{1}(t)\right)\right) \\
F_{m}(t)+F_{h}(t)
\end{array}\right), \quad z_{m e s}(t)=\left(\begin{array}{c}
\theta_{s}(t)-\theta_{e}(t) \\
\theta_{e}(t)-\theta_{m}(t)
\end{array}\right) .
$$

So we can get:

$$
\begin{aligned}
& A_{\text {mes }}^{0}=\left(\begin{array}{ccccc}
A_{s}-B_{s} K_{0}^{s}-B_{s} K_{1} & -B_{s} K_{2} & 0 & -B_{s} K_{3} & 0 \\
0 & A_{m}-B_{m} K_{0}^{m} & 0 & 0 & 0 \\
0 & 0 & A_{m}-B_{m} K_{0}^{m} & 0 & 0 \\
1 & -1 & 0 & 0 & 0 \\
0 & 1 & -1 & 0 & 0
\end{array}\right)
\end{aligned}
$$

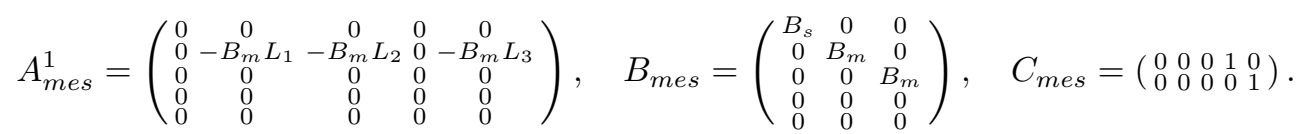

By Corollary 2.2, we can verify the global stability of the system with the force tracking $F_{m}(t)=F_{e}\left(t-\tau_{2}(t)\right)$, in which the delay $\tau_{2}(t)$ can not be avoided. Note that in this paper, the position tracking and the force tracking are assured respectively from master to slave and from slave to master.

Remark 4: The global design of $L$ and $K$ cannot be achieved in a single LMI problem. The main reason is that transformations like (33) cannot be applied on both gains at the same time. 
Table 1. Admissible delay upper bound $h_{2}$ for various $h_{1}$ and $\mu=0.3$

\begin{tabular}{lllllll}
\hline method & $h_{1}$ & 1.0 & 2.0 & 3.0 & 4.0 & 5.0 \\
\hline He et al. 2007 & $h_{2}$ & 2.21 & 2.40 & 3.33 & 4.27 & 5.23 \\
Shao 2009 & $h_{2}$ & 2.24 & 2.47 & 3.38 & 4.32 & 5.27 \\
Theorem 2.1 & $h_{2}$ & 2.35 & 2.58 & 3.47 & 4.39 & 5.33 \\
\hline
\end{tabular}

\section{$5 \quad$ Numerical examples}

We consider a numerical example from (He et al. 2007, Shao 2009) to show the reduced conservatism of our delay-scheduled LKF-based stability criteria.

- Example: Consider the system (1) without the perturbation $w(t)$ and:

$$
A_{0}=\left(\begin{array}{cc}
0 & 1 \\
-1 & -2
\end{array}\right), \quad A_{1}=\left(\begin{array}{cc}
0 & 0 \\
-1 & 1
\end{array}\right) \text {. }
$$

For $\mu=0.3$ and unknown $\mu$, the admissible delay upper bounds $h_{2}$ are shown in Table 1 .

Remark 5: The result of (Park et al. 2011) get similar admissible delay upper bound as Theorem 2.1 but as specified in Remark 2, (Park et al. 2011) is not suitable for control design.

\section{Illustrative example}

In this section, some simulations are performed in different working conditions to evaluate the performance of the proposed approach and compare it to other results ((Ye et al. 2009, 2011) power-based time domain passivity, (Nuño et al. 2009) P-like controller, (Hua and Liu 2010) LKF-based controllers). These simulation are chosen to compare the quality of the position tracking in two situations: change of position of the master in free motion and in wall contact.

The model chosen for the simulations is the one of our experimental platform:

$$
\begin{aligned}
& A_{m}=-\frac{1}{\tau_{m}}, \quad B_{m}=\frac{K_{m}}{\tau_{m}}, \quad \tau_{m}=0.448, \quad K_{m}=0.0176, \\
& A_{s}=-\frac{1}{\tau_{s}}, \quad B_{s}=\frac{K_{s}}{\tau_{s}}, \quad \tau_{s}=0.3125, \quad K_{s}=3.66 .
\end{aligned}
$$

The minimum and maximum value of time-varying delays are taken as $h_{1}=0.001 \mathrm{~s}$ and $h_{2}=0.2 s$ (greater allowable maximum delays can also be handled), which satisfy many networkbased applications of teleoperation such as internet-based teleoperation. For the simulation of asymmetric time-varying delays in the two channels, we use a band-limited white noise.

As stated previously, a velocity feedback is needed in this control structure (Figure 2). Let consider that it is design to get $[-5.0]$ for the master's pole, and $[-100.0]$ for the slave. It is achieved by choosing $K_{0}^{m}=70.455$ and $K_{0}^{s}=8.265$. Applying the design procedure described in the previous section, one get the following gains and performances:

$$
\begin{array}{ll}
L=(75.4709-75.4727485 .1147), & \gamma_{m i n}^{L}=0.0048, \\
K=(-2.2847-5.8599329 .6448), & \gamma_{m i n}^{K}=0.0166 .
\end{array}
$$

From Corollary 2.2, the global stability of the system is verified with $\gamma_{\min }^{g}=0.0042$ which should ensure a good position tracking.

\subsection{Tracking in abrupt tracking motion}

With the same simulation condition, our objective is to show the system stability and compare the position tracking and convergence performance. 


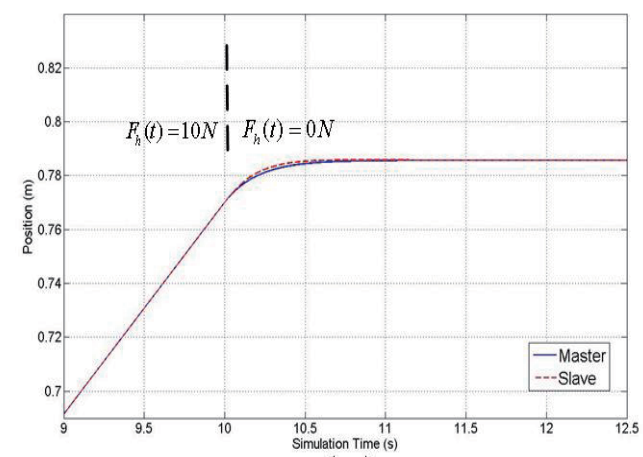

(a)

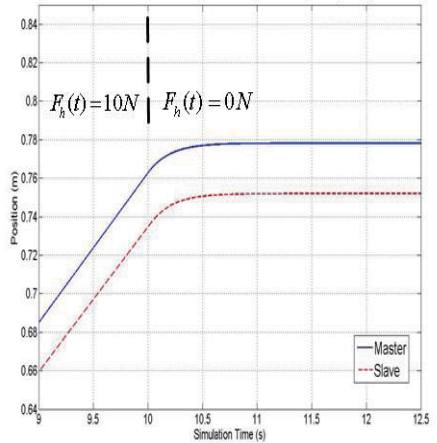

(c)

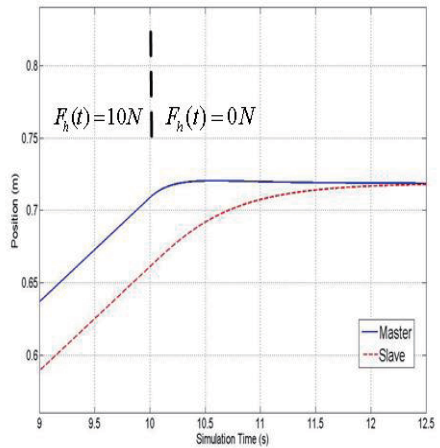

(d)

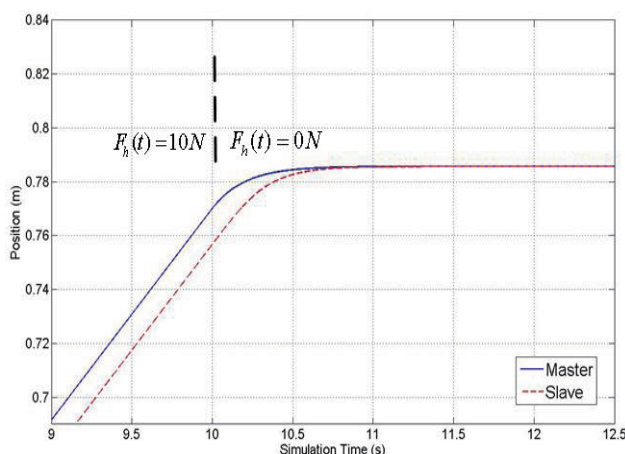

(b)

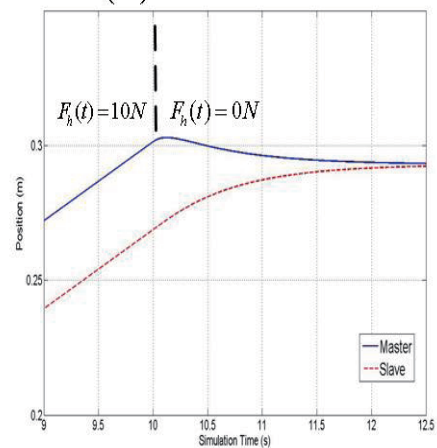

(e)

Figure 3. Position response in abrupt tracking motion ((a): force-reflecting emulator control scheme; (b): force-reflecting emulator control scheme without emulator; (c): from (Ye et al. 2009); (d): from (Nuño et al. 2009); (e): from (Hua and Liu 2010)).

In Figure 3, (a) shows the result of the force-reflecting emulator control scheme, and for the comparison reasons, the result of the same control scheme but without the emulator is shown in (b), in this case, the controller $C$ is designed under the same procedure proposed before. We can see that, the emulator of master is important for improving the position tracking. The passivity-based position tracking of (Ye et al. 2009, Nuño et al. 2009) is presented in (c) and (d). (e) shows the result of (Hua and Liu 2010), which also used Lyapunov-Krasovskii functionals to design the controllers and analyze the stability conditions. In (Hua and Liu 2010), the controller gains should be determined before, and then, the stability analysis and allowable maximum time delays will be checked by LKF theorems in form of LMI. We have resolved this problem in this paper, our controllers can be obtained directly by LKF theorems.

It is clear that our method achieves the stability and the position tracking. Concerning (Ye et al. 2009, Nuño et al. 2009, Hua and Liu 2010), the position convergence is ensured, but there is still a position deviation between the master and the slave.

\subsection{Tracking in wall contact motion}

In this case, the slave robot is driven to a hard wall with a stiffness of $K_{e}=30 \mathrm{kN} / \mathrm{m}$ located at $x \geq 1.0 \mathrm{~m}$. Our aim is to show that, by our method: 1 ) when the slave robot reaches the wall, the master robot stops as quickly as possible; 2) when the slave robot returns after hitting the wall $\left(F_{e}(t)=0\right)$, the system restores the position tracking between the master and slave robots; 3 ) when the slave is in contact with the wall, the force tracking from slave to master can be assured.

In Figure 4, our scheme can ensure the points 1) and 2) as mentioned above and get a better position performance. By the approach in (Ye et al. 2009), the position deviation between the master and the slave is larger, and when the slave robot returns from the wall, the teleoperation system can not restore the position tracking. (Hua and Liu 2010) can also ensure the points 1) 


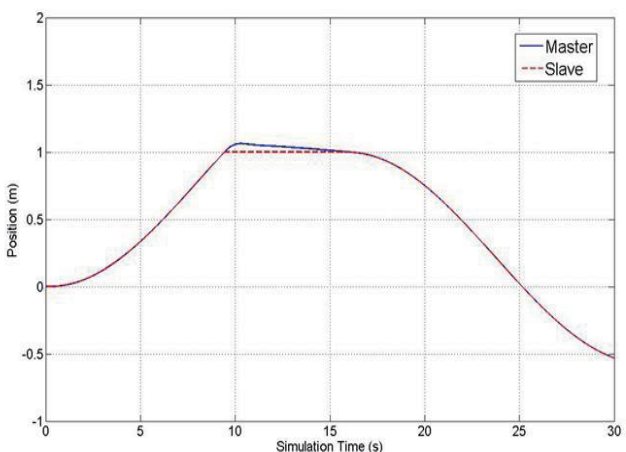

(a)

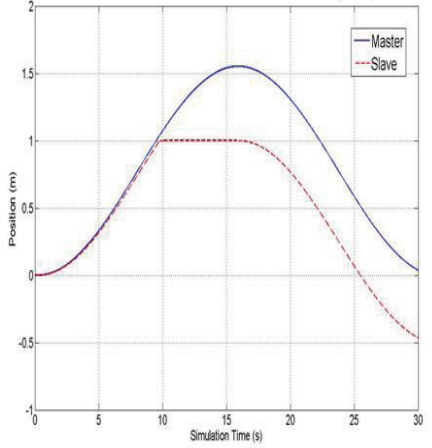

(c)

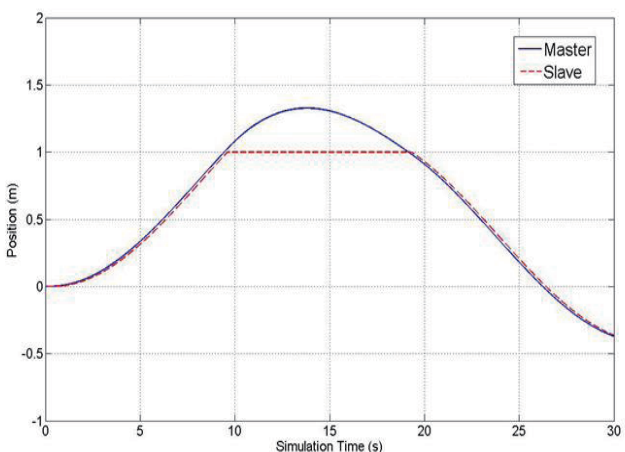

(b)

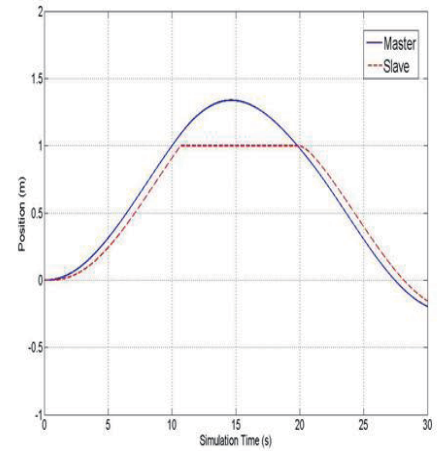

(d)

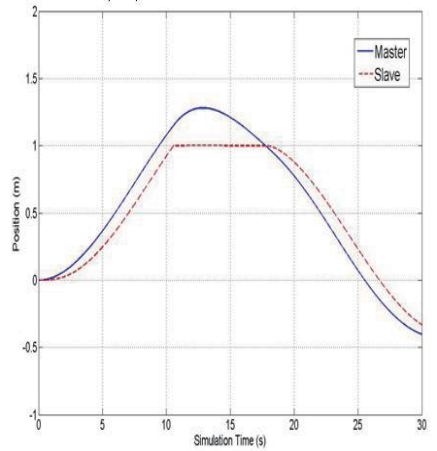

(e)

Figure 4. Position response in wall contact motion ((a): force-reflecting emulator control scheme; (b): force-reflecting emulator control scheme without emulator; (c): from (Ye et al. 2009); (d): from (Nuño et al. 2009); (e): from (Hua and Liu 2010)).

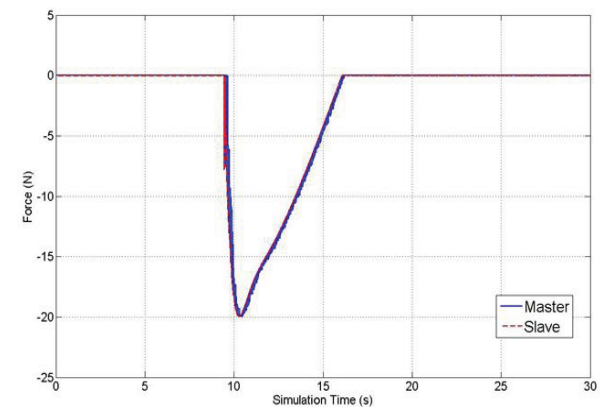

Figure 5. Force response in wall contact motion $\left(F_{m}(t) ; F_{e}(t)\right)$.

and 2), but compared with the approach in (Hua and Liu 2010), because we have introduced $H_{\infty}$ control theory, our architecture can get a better position tracking and faster position convergence.

Figure 5 shows the force tracking from the slave to the master, $F_{m}(t)$ and $F_{e}(t)$, which satisfies $3)$. We added a simple perturbation observer in the force-reflecting emulator control scheme to obtain unmeasured $F_{e}(t)$.

In the case of 'large' delays, we suppose the allowable maximum time-varying delays, $h_{2}=$ $1.0 s$, by the theorems in the paper, the controllers are recalculated. The simulation results are presented in Figure 6, we can see that, our method can support 'large' delays, while ensuring stability and performance. 


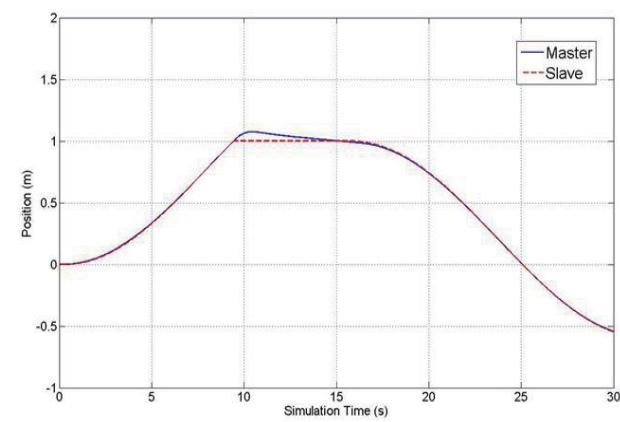

(a)

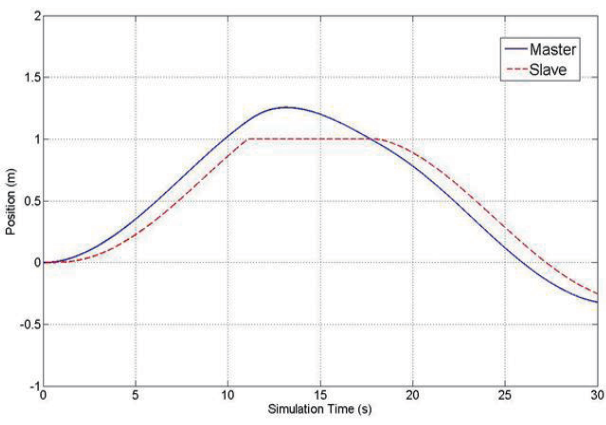

(b)

Figure 6. Position response with 'large' delays in wall contact motion ((a): force-reflecting emulator control scheme; (b): from (Hua and Liu 2010)).

\section{Conclusions}

This paper mainly presented two contributions: from the point view of time delay systems, the delay-scheduled LKF-based criteria improved over some previous ones, in which the computed admissible upper bound of time delay is larger, it is also worthwhile to point out that our method is efficient to design the controllers of delayed teleoperation, which is the second contribution in this paper; from the point view of delayed teleoperation, combining the theories of delayscheduled LKF and $H_{\infty}$ allowed us to propose a new bilateral control scheme for position and force tracking, it ensures asymptotic stability and guaranteed performance under asymmetric time-varying network delays. The emulator of master and controller $C$ are computed in terms of LMI. The new architecture, which makes use of the " emulator"' of master in the teleoperation, is a comprehensive summary of the position-position and position-force schemes.

The simulations achieved by YALMIP and SIMULINK demonstrated the effectiveness and benefits of the delay-scheduled LKF-based stability results, and presented that the teleoperation system designed based on this new controller is efficient in different working conditions. The performance comparison with other recent approaches has been achieved.

\section{Acknowledgements}

The authors would like to acknowledge Prof. Romeo Ortega for his valuable comments on this work.

\section{References}

Anderson, R.W., and Spong, M.W. (1989), "Bilateral Control of Teleoperators with Time Delay," IEEE Trans. Automat. Contr., 34, 494-501.

Arcara, P., and Melchiorri, C. (2002), "Control Schemes for Teleoperation with Time Delay: a Comparative Study," Robotics and Autonomous Systems, 38, 49-64.

Chopra, N., Berestesky, P., and Spong, M.W. (2008), "Bilateral Teleoperation over Unreliable Communication Networks," IEEE Trans. Contr. Syst. Technol., 16, 304-313.

Chopra, N., Spong, M.W., Ortega, R., and Barabanov, N.E. (2006), "On Tracking Performance in Bilateral Teleoperation," IEEE Trans. Robot., 22, 861-866.

Daly, J.M., and Wang, D.W.L. (2010), "Time-Delayed Bilateral Teleoperation with Force Estimation for n-DOF Nonlinear Robot Manipulators," in Proc. IEEE/RSJ Int. Conf. on Intelligent Robots and Systems (IROS'10), October, Taipei, Taiwan, pp. 3911-3918.

Delgado, E., and Barreiro, A. (2009), "Stability of Teleoperation Systems for Time-Varying 
Delays by Lyapunov-Krasovskii and Frequencial Techniques," in Proc. Annual Conf. of IEEE on Industrial Electronics (IECON'09), November, Porto, Portugal, pp. 1748-1753.

Fridman, E. (2006), "A New Lyapunov Technique For Robust Control of Systems with Uncertain Non-Small Delays," IMA J. Math. Contr. Info., 23, 165-179.

Fridman, E., and Shaked, U. (2001), "New Bounded Real Lemma Representations for TimeDelay Systems and Their Applications," IEEE Trans. Automat. Contr., 46, 1973-1979.

Garcia-Valdovinos, L.G., Parra-Vega, V., and Arteaga, M.A. (2007), "Observer-Based Sliding Mode Impedance Control of Bilateral Teleoperation under Constant Unknown Time Delay," Robot. Auton. Syst., 55, 609-617.

Gu, K., Kharitonov, V.L., and Chen, J. (2003)Stability of Time-Delay Systems, Boston, Basel, Berlin: Birkhuser.

He, Y., Wang, Q.G., Lin, C., and Wu, M. (2007), "Delay-Range-Dependent Stability for Systems with Time-Varying Delay," Automatica, 43, 371-376.

Hou, Z.L., Wei, Q., Li, F., and Han, D.P. (2010), "Design on a Half Closed-Loop Bilateral Teleoperation System," in Proc. Chinese Control Conference (CCC'10), July, Beijing, China, pp. 3432-3436.

Hua, C.C., and Liu, X.P. (2010), "Delay-Dependent Stability Criteria of Teleoperation Systems with Asymmetric Time-Varying Delays," IEEE Trans. Robot., 26, 925-932.

Hua, C.C., and Liu, X.P. (2011), "Teleoperation over the Internet with/without Velocity Signal," IEEE Trans. Instrum. Meas., 60, 4-13.

Jiang, W.J., Kruszewski, A., Richard, J.P., and Toguyeni, A. (2008), "A Gain Scheduling Strategy for the Control and Estimation of a Remote Robot via Internet," in Proc. Chinese Control Conference (CCC'08), July, Kunming, China, pp. 793-799.

Kruszewski, A., Jiang, W.J., Fridman, E., Richard, J.P., and A.Toguyeni, (2011), "A Switched System Approach to Exponential Stabilization through Communication Network," IEEE Trans. Contr. Syst. Technol., to appear.

Natori, K., Tsuji, T., Ohnishi, K., Hace, A., and Jezernik, K. (2010), "Time-Delay Compensation By Communication Disturbance Observer for Bilateral Teleoperation Under Time-Varying Delay," IEEE Trans. Ind. Electron., 57, 1050-1062.

Nuño, E., Basañez, L., and Ortega, R. (2011), "Passivity-Based Control for Bilateral Teleoperation: a Tutorial," Automatica, 47, 485-495.

Nuño, E., Basañez, L., Ortega, R., and Spong, M.W. (2009), "Position Tracking for Non-Linear Teleoperators with Variable Time Delay," Int. J. Robot. Res., 28, 895-910.

Park, P., Kob, J., and Jeong, C. (2011), "Reciprocally Convex Approach to Stability of Systems with Time-Varying Delays," Automatica, 47, 235-238.

Polushin, I.G., Liu, P.X., and Lung, C.H. (2007), "A Force-Reflection Algorithm for Improved Transparency in Bilateral Teleoperation with Communication Delay," IEEE/ASME Trans. Mechatronics, 12, 361-374.

Richard, J.P. (2003), "Time Delay Systems: an Overview of Some Recent Advances and Open Problems," Automatica, 39, 1667-1694.

Ryu, J.H., Preusche, C., Hannaford, B., and Hirzinger, G. (2005), "Time Domain Passivity Control with Reference Energy Following," IEEE Trans. Contr. Syst. Technol., 13, 737-742.

Shahbazi, M., Talebi, H., and Yazdanpanah, M. (2010), "A Control Architecture for Dual User Teleoperation with Unknown Time Delays: a Sliding Mode Approach," in Proc. IEEE/ASME Int. Conf. on Advanced Intelligent Mechatronics (AIM'10), July, Montral, Canada, pp. 12211226.

Shao, H. (2009), "New Delay-Dependent Stability Criteria for Systems with Interval Delay," Automatica, 45, 744-749.

Smith, A.C., and Hashtrudi-Zaad, K. (2005), "Neural Network-Based Teleoperation using Smith Predictors," in Proc. IEEE Int. Conf. on Mechatronics 85 Automation (ICMA'05), July, Niagara Falls, Canada, pp. 1654-1659.

Tadmor, G. (2000), "The Standard $H_{\infty}$ Problem in Systems with a Single Input Delay," IEEE 
Trans. Automat. Contr., 45, 382-397.

Witrant, E., Canudas-De-Wit, C., Georges, D., and Alamir, M. (2007), "Remote Stabilization via Communication Networks with a Distributed Control Law," IEEE Trans. Automat. Contr., $52,1480-1485$.

Xu, S.Y., Lam, J., and Zou, Y. (2006), "New Results on Delay-Dependent Robust $H_{\infty}$ Control for Systems with Time-Varying Delays," Automatica, 42, 343-348.

Ye, Y.Q., Pan, Y.J., and Gupta, Y. (2009), "Time Domain Passivity Control of Teleoperation Systems with Random Asymmetric Time Delays," in Proc. IEEE Conf. on Decision and Control, and Chinese Control Conference (CDC'09, CCC'09), December, Shanghai, China, pp. $7533-7538$.

Ye, Y.Q., Pan, Y.J., Gupta, Y., and Ware, J. (2011), "A Power-Based Time Domain Passivity Control for Haptic Interfaces," IEEE Trans. Contr. Syst. Technol., 19, 874-883.

Zampieri, S. (2008), "Trends in Networked Control Systems," in Proc. World Congress the International Federation of Automatic Control (IFAC'08), July, Seoul, Korea, pp. 2886-2894.

Zhang, B., Kruszewski, A., and Richard, J.P. (2011), "A Novel Control Scheme for Teleoperation with Guaranteed Performance under Time-Varying Delays," in Proc. Chinese Control and Decision Conference (CCDC'11), May, Mianyang, China.

Zhang, X.M., and Han, Q.L. (2008), "Robust $H_{\infty}$ Filtering for a Class of Uncertain Linear Systems with Time-Varying Delay," Automatica, 44, 157-166. 